\title{
The effect of tennis match play on joint range of motion in junior players
}

\author{
Rok Šrimpf ${ }^{1}$, Tjaša Filipčič², and Aleš Filipčič ${ }^{1, *}$ \\ ${ }^{1}$ Faculty of Sport, University of Ljubljana, Ljubljana, Slovenia; and ${ }^{2}$ Faculty of Education, University of Ljubljana, \\ Ljubljana, Slovenia
}

Copyright: (C) 2019 R. Šrimpf et al. This is an open access article licensed under the Creative Commons Attribution License (https://creativecommons.org/licenses/by/4.0/).

\begin{abstract}
Background: Tennis players perform a large number of multidirectional and cutting movements, together with asymmetrical rotational motions resulting from serves and groundstrokes. Numerous shot repetitions and multidirectional motions directly influence a tennis player's upper and lower extremity joint range of motion (ROM). Objective: This study evaluated the changes in the range of motion of junior tennis players before and after match play. Methods: Twelve male (age $14.4 \pm 1.3$ years) and twelve female (age $13.4 \pm 2.1$ years) junior tennis players participated in the study. Two skilled experts performed 13 tests to measure passive range of motion of the dominant and non-dominant shoulder, elbow, wrist, hip, knee, and subtalar joints, before and after match play. The $t$-test and Wilcoxon test were used to determine the differences between the ROM before and after the tennis match, and the differences between the dominant and non-dominant sides of the body. Results: Bilateral measurement of the internal rotation of the shoulder joints, forearm pronation, and inversion of the subtalar joints before match play, show significant differences between extremities, similarly as the elevation of the arm in the coronal plane and forearm pronation, after a tennis match, were also found to display statistically significant differences. ROM values were higher for the internal rotation of both shoulders, external rotation of the non-dominant shoulder, elevation of the arms in the coronal plane, flexion in the elbow joints, pronation in the forearms, adduction in the hips, as well as eversion and inversion of the subtalar joints. Conclusions: Male and female junior tennis players increase their joint ROM during match play through motions which are involved in the execution of tennis shots and tennis movement patterns. Tennis matches that last 90 minutes or less do not have a negative impact on the flexibility of young tennis players.
\end{abstract}

Keywords: racket sports, youth, goniometer, flexibility

\section{Introduction}

In tennis, it is widely accepted that success requires a great deal of training and frequent participation in competitions in all age categories (Reid, Crespo, Santilli, Miley, \& Dimmock, 2007; Reid \& Morris, 2011). Kovacs (2006) found that the physical demands of tennis match play cause musculoskeletal adaptations that are sometimes positive (increased strength) and sometimes negative (decreased joint range of motion and reduced muscular flexibility). Gillet, Begon, Sevrez, Berger-Vachon, and Rogowski (2017) note that the range of motion (ROM) of glenohumeral joints in young tennis players decreases with age, while absolute power increases. The optimal ratio between flexibility

\footnotetext{
* Address for correspondence: Aleš Filipčič, Faculty of Sport, University of Ljubljana, Gortanova 22, 1000 Ljubljana, Slovenia. E-mail: ales.filipcic@fsp.uni-lj.si
}

and strength is thought to be crucial in protecting the shoulder complex during intensive overhead motions. Lack of flexibility in athletes has been linked to a decrease in performance and increase in muscular injuries (Shellock \& Prentice, 1985).

During a tennis match, players usually perform a large number of multidirectional and cutting movements along with asymmetric rotational motions resulting from the execution of a serve or groundstroke (Roetert, Kovacs, Knudson, \& Groppel, 2009). Tennis match load is expressed as the number of shots a player makes and the distance covered while performing movement patterns that include fast starts, directional changes, stops, and jumps. Weber (2001) reported that in an average rally, professional players hit three shots and cover $3 \mathrm{~m}$ for each contact with the ball, which amounts to approximately 1,000 executed shots and $3 \mathrm{~km}$ of distance covered during a best of three sets match. Kovacs (2006) found that all movements 
directly influence the upper and lower limb joints' range of motion in tennis players.

Effective stroke execution in tennis maximally utilizes the entire kinetic chain. Coordinated use of specific body segments (legs, hips, trunk, arms) in rhythmical movements allows tennis players to adapt to different game situations and perform strokes with controlled, optimal variation. Tennis strokes (serve, forehand, backhand) are performed as throwing motions, where lower body force production is transferred up into the upper body and out through the racket into the ball (Kibler \& Chandler, 2003).

Previous studies have analyzed flexibility (Chandler et al., 1990) and the glenohumeral joint ROM (Ellenbecker \& Roetert, 2003; Ellenbecker, Roetert, Piorkowski, \& Schulz, 1996; Gillet, Begon, Diger, Berger-Vachon, \& Rogowski, 2018; Schmidt-Wiethoff, Rapp, Mauch, Schneider, \& Appell, 2004; Stanley, McGann, Hall, McKenna, \& Brigfa, 2003). Due to numerous shot repetitions, especially pertaining to the serve, there is often a reported deficit in glenohumeral internal rotation ROM of the dominant arm of tennis players (Ellenbecker, Roetert, Bailie, Davies, \& Brown, 2002; Kibler, Chandler, Livingston, \& Roetert, 1996; Moreno-Pérez, Moreside, Barbado, \& Vera-Garcia, 2015; Roetert, McCormick, Brown, \& Ellenbecker, 1996). Similarly, Martin, Kulpa, Ezanno, Delamarche, and Bideau (2016) found a significant decrease in passive shoulder internal rotation and total ROM during a 3-hour tennis match. Elite junior tennis players have a relative muscular strength imbalance between the internal and external shoulder rotators of the dominant arm (Ellenbecker \& Roetert, 2003). Junior tennis players have a stronger horizontal abduction and adduction in their dominant versus their non-dominant shoulder (Silva et al., 2006).

Few studies have dealt with the influence of tennis match play on ROM of the lower extremities. Young et al. (2014) assessed hip range of motion and its association with injury but was unable to identify any specific side-to-side rotational adaptation in the dominant or non-dominant hip of female tennis players. MorenoPérez, Ayala, Fernandez-Fernandez, and Vera-Garcia (2016) found that male and female tennis players had restricted mobility measures of hip flexion, extension, and abduction. At the same time, they pointed out that asymmetric hip joint ROM measurements may indicate abnormalities and the need for flexibility training.

The use of goniometric measurement of joint range of motion is a widespread practice in orthopedic and sports physical therapy (Norkin \& White, 2016). An objective range of motion measurement is a necessary part of player evaluation, as well as demonstration of injury treatment efficacy. Rothstein, Miller, and
Roettger (1983) studied goniometric measurements of the knee and elbow and found high intratester and intertester reliability in the clinical setting. The reliability of goniometric measurements of joint motion has been extensively studied by Horger (1989), who indicated that measurements of wrist motion were highly reliable. Riddle, Rothstein, and Lamb (1987) found suitable reliability of goniometric ROM measurements of the shoulders. Watkins, Riddle, and Lamb (1991) suggested the use of a goniometer to make repeated ROM measurements of the knee to minimize the error associated with these measurements.

Previous studies of ROM among tennis players have focused mainly on shoulder and hip joints (Vad, Gebeh, Dines, Altchek, \& Norris 2003) while studies that focused on other joints (wrist and ankle) were rare (Kibler \& Chandler, 2003). Chiang, Hsu, Chiang, Chang, and Tsai (2016) compared the internal and external rotation of the dominant and non-dominant shoulders of junior female tennis players. The internal rotation of the dominant shoulder was significantly smaller than that of the non-dominant shoulder. Flexibility of the glenohumeral internal rotation may be a factor affecting performance in juniors. Kovalchik and Reid (2017) believe that physical loads, in professional, as well as junior tennis, are more and more demanding. Movements are faster and more diverse, and tennis strokes are executed in dynamic conditions with larger amplitudes. With this in mind, the present study aimed to use goniometric measurements to assess how the range of motion in the dominant and non-dominant shoulder, elbow, wrist, hip, knee, and ankle, changes, before and after junior match play.

\section{Methods}

\section{Participants}

Twelve male $(14.4 \pm 1.3$ years, $165.7 \pm 13.8 \mathrm{~cm}, 57.1 \pm 14.6$ $\mathrm{kg})$ and twelve female junior tennis players (13.4 \pm 2.1 years, $156.7 \pm 9.4 \mathrm{~cm}, 45.5 \pm 14.3 \mathrm{~kg}$ ) volunteered to participate in the study. Participants were recruited from five tennis clubs, all were right-handed and used a two-handed backhand stroke. The study was conducted during the pre-competitive season. All players were in regular training and were ranked on the national ranking list. Five players also had an ITF junior ranking. Subjects were free from any upper and lower extremity injury.

\section{Measurement}

A standard plastic universal goniometer (66fit Goniometer - $30 \mathrm{~cm}$, 66fit, Spalding, United Kingdom) was used for measurement, with scales marked in one-degree increments. Prior to participation, the 
experimental procedures and potential risks were explained to the legal representatives of participants in full, and all signed written informed consent. The study was approved by the University Office for Research Ethics and conformed to the Declaration of Helsinki.

The testing procedure was standardized and undertaken in the following order. On arrival, each participant completed a questionnaire pertaining to age, dominant playing hand, level of play, duration of training and competition attendance, starting age, and history of injuries. All participants performed a 20-minute warm-up, which included running, dynamic warm-up exercises, and athletic activation movement tasks. This was followed by measurements of body weight and height. Two skilled experts (physiotherapists) measured the following movements of the tennis players before and after the tennis match:

- internal rotation in the shoulder joint - shoulder internal rotation,

- external rotation in the shoulder joint - shoulder external rotation,

- elevation of the arm in the coronal plane - arm elevation,

- flexion in the elbow joint - elbow flexion,

- pronation of the forearm - forearm pronation,

- supination of the forearm - forearm supination,

- dorsal flexion (extension) in the wrist joint - wrist dorsal flexion,

- volar flexion in the wrist joint - wrist volar flexion,

- abduction in the hip joint - hip abduction,

- adduction in the hip joint - hip adduction,

- flexion in the knee joint - knee flexion,

- eversion (pronation) in the subtalar joint - subtalar eversion,

- inversion (supination) in the subtalar joint - subtalar inversion.

Passive range of motion was measured as the maximal range of joint motion attained by a participant during the application of external force (Horger, 1989). Measurements were performed according to protocols by Jakovljević and Hlebš (2017). The task of the first examiner was to perform a specific motion on the participant's body in accordance with instructions laid out by the protocol, while the second examiner then measured the amplitude of the movement in each individual joint. A 30-second rest period was given between trials, limbs, and tests. Each measurement was performed twice and the higher score was included in the analysis. Following the completion of the measurements, the participant played an unofficial training tennis match, which lasted 90 minutes. Workload of the tennis match was monitored, using a wearable device (Armbeep Wrist Monitoring Device 1.0, Biometrika, Maribor,
Slovenia) and system for movement and biometric data acquisition (Kos \& Kramberger, 2017). The device monitored and collected the following parameters: the number of shots, wrist speed, and acceleration in the contact zone. After the tennis match was concluded, we repeated the measurement of all 13 tests for passive range of motion on both sides of the participant's body. All ROM tests were performed after the tennis match (12.6 \pm 8.5 minutes) in a closed environment where the temperature was stable for the duration of the tests.

\section{Statistical analyses}

The means and standard deviations were calculated for all variables. The $t$-test for dependent variables was used to determine the differences between the range of motion before and after the tennis match, and the differences between the dominant and nondominant sides of the body. Before determining these differences, we tested the assumption of normal distribution (Shapiro-Wilk test). When this assumption was violated, we used the Wilcoxon test. A result was deemed statistically significant when $p \leq .05$. Statistical analysis was done using IBM SPSS (Version 24; IBM, Armonk, NY, USA).

\section{Results}

During the match, tennis players performed $522.3 \pm 53.6$ shots in $127.5 \pm 24.3$ rallies, which, on average, computes to $4.5 \pm 1.1$ shots in an individual rally.

The results obtained in this study showed only statistically significant bilateral differences before the match for internal rotation in the shoulder joint (higher ROM was found in the non-dominant joint), forearm pronation and inversion in the subtalar joints (in both variables higher ROM values were observed in the dominant joint), see Table 1. After the match (Table 2) statistically significant bilateral differences were observed in elevation of the arm in the coronal plane and forearm pronation (higher ROM values in the dominant joint for both variables).

The comparison of ROM before and after the match showed that the differences in internal shoulder rotation, arm elevation, elbow flexion, forearm pronation, hip adduction, subtalar eversion, and inversion in both joints after the match were significant (Table 3). Significant differences were also shown in external shoulder rotation of the non-dominant shoulder.

The results reveal a significant increase of range of motion in the internal rotation of the dominant and non-dominant shoulder. The same was found in the external rotation of the non-dominant shoulder. 
Table 1

Differences in range of motion between the dominant and non-dominant joint before match play. Values are presented in degrees as mean $\pm S D$.

\begin{tabular}{lcccc}
\hline Variable & Dominant & Non-dominant & $t$ & $p$ \\
\hline Internal rotation in the shoulder joint & $59.5 \pm 8.2$ & $63.7 \pm 7.2$ & -3.32 & $.005^{1}$ \\
External rotation in the shoulder joint & $83.3 \pm 16.5$ & $83.2 \pm 10.5$ & 0.04 & .967 \\
Elevation of the arm in the coronal plane & $120.2 \pm 26.7$ & $116.6 \pm 25.1$ & 1.89 & .075 \\
Flexion in the elbow joint & $152.2 \pm 3.2$ & $152.1 \pm 5.3$ & -0.19 & .847 \\
Pronation in the forearm & $81.8 \pm 5.8$ & $78.2 \pm 6.7$ & -3.18 & $.005^{1}$ \\
Supination in the forearm & $83.4 \pm 5.3$ & $84.7 \pm 3.8$ & -1.52 & .146 \\
Dorsal flexion in the wrist joint & $81.8 \pm 5.5$ & $82.9 \pm 7.4$ & -0.87 & .393 \\
Volar flexion in the wrist joint & $83.3 \pm 9.2$ & $81.7 \pm 6.8$ & 1.17 & .255 \\
Abduction in the hip joint & $49.3 \pm 11.6$ & $49.4 \pm 10.2$ & -0.11 & .909 \\
Adduction in the hip joint & $21.3 \pm 4.4$ & $20.7 \pm 4.6$ & 0.71 & .493 \\
Flexion in the knee joint & $157.9 \pm 9.0$ & $159.1 \pm 6.6$ & -1.51 & .148 \\
Eversion in the subtalar joint & $6.9 \pm 1.7$ & $7.1 \pm 1.6$ & -0.40 & .698 \\
Inversion in the subtalar joint & $33.4 \pm 5.9$ & $29.9 \pm 5.8$ & 2.44 \\
\hline
\end{tabular}

Note. ${ }^{1} t$-test showed significantly different value between the dominant and non-dominant joint $(p \leq .05)$.

Table 2

Differences in range of motion between the dominant and non-dominant joint after match play. Values are presented in degrees as mean $\pm S D$.

\begin{tabular}{|c|c|c|c|c|}
\hline Variable & Dominant & Non-dominant & $t$ & $p$ \\
\hline Internal rotation in the shoulder joint & $67.4 \pm 5.6$ & $69.7 \pm 5.9$ & -1.53 & .144 \\
\hline External rotation in the shoulder joint & $88.9 \pm 4.5$ & $88.2 \pm 5.3$ & 0.80 & .433 \\
\hline Elevation of the arm in the coronal plane & $134.1 \pm 23.6$ & $124.3 \pm 22.0$ & 5.04 & $<.001^{2}$ \\
\hline Flexion in the elbow joint & $156.8 \pm 3.8$ & $156.9 \pm 4.1$ & -0.06 & .949 \\
\hline Pronation in the forearm & $84.3 \pm 4.6$ & $81.6 \pm 7.0$ & 2.79 & $<.001^{1}$ \\
\hline Supination in the forearm & $84.9 \pm 4.0$ & $86.3 \pm 2.5$ & -1.82 & .086 \\
\hline Dorsal flexion in the wrist joint & $83.0 \pm 10.5$ & $85.1 \pm 8.9$ & -1.57 & .134 \\
\hline Volar flexion in the wrist joint & $84.0 \pm 4.7$ & $83.6 \pm 7.7$ & 0.31 & .759 \\
\hline Abduction in the hip joint & $52.9 \pm 9.9$ & $51.1 \pm 7.5$ & 0.74 & .465 \\
\hline Adduction in the hip joint & $24.9 \pm 4.5$ & $23.7 \pm 3.5$ & 1.53 & .143 \\
\hline Flexion in the knee joint & $159.4 \pm 6.1$ & $159.4 \pm 6.3$ & 0.00 & $>.99$ \\
\hline Eversion in the subtalar joint & $10.4 \pm 2.3$ & $10.9 \pm 2.6$ & -0.92 & .368 \\
\hline Inversion in the subtalar joint & $37.2 \pm 4.9$ & $35.2 \pm 5.1$ & 1.83 & .083 \\
\hline
\end{tabular}

Note. $\quad{ }^{1} t$-test showed significantly different value between the dominant and non-dominant joint $(p \leq .05) .{ }^{2}$ Wilcoxon test showed significantly different value between the dominant and non-dominant joint $(p \leq .05)$.

A significant increase of ROM was also observed in the elevation of both arms in the coronal plane. The range of motion of the dominant shoulder in elevation in the coronal plane increased by 14 degrees after the match. No significant differences were detected in the external rotation of the dominant shoulder.

Significant differences were also observed in the flexion of both elbow joints and pronation in both forearms. Differences in ROM values in flexion and pronation of both elbows amounted to an increase of 3-4 degrees, after the match. There were no significant differences found in the supination of the forearms, or in the dorsal and volar flexion of the wrist joints of both limbs.

The changes of ROM before and after the tennis match were significant in the adduction of both hip joints, and in the eversion and inversion of both subtalar joints. No statistically significant differences were observed in the abduction of either hip joint, or the flexion in either knee joint. 
Table 3

Range of motion before and after the match in the dominant and non-dominant shoulder, elbow, wrist, hip, knee, and ankle. Values are presented in degrees as mean $\pm S D$.

\begin{tabular}{|c|c|c|c|c|}
\hline Variable & Before the match & After the match & $t$ & $p$ \\
\hline Internal rotation in the dominant shoulder joint & $59.5 \pm 8.2$ & $67.4 \pm 5.6$ & -4.86 & $<.001^{1}$ \\
\hline Internal rotation in the non-dominant shoulder joint & $63.7 \pm 7.2$ & $69.7 \pm 5.9$ & -5.64 & $<.001^{1}$ \\
\hline External rotation in the dominant shoulder joint & $83.3 \pm 16.5$ & $88.9 \pm 4.5$ & -1.47 & .160 \\
\hline External rotation in the non-dominant shoulder joint & $83.2 \pm 10.5$ & $88.2 \pm 5.3$ & -2.62 & $.018^{1}$ \\
\hline Elevation of the dominant arm in the coronal plane & $120.2 \pm 26.7$ & $134.1 \pm 23.6$ & -4.40 & $.006^{1}$ \\
\hline Elevation of the non-dominant arm in the coronal plane & $116.6 \pm 25.1$ & $124.3 \pm 22.0$ & -3.16 & $<.001^{1}$ \\
\hline Flexion in the dominant elbow joint & $152.2 \pm 3.2$ & $156.8 \pm 3.8$ & -6.00 & $<.001^{1}$ \\
\hline Flexion in the non-dominant elbow joint & $152.1 \pm 5.3$ & $156.9 \pm 4.1$ & -5.76 & $<.001^{1}$ \\
\hline Pronation in the dominant forearm & $81.8 \pm 5.8$ & $84.3 \pm 4.6$ & -2.76 & $<.001^{1}$ \\
\hline Pronation in the non-dominant forearm & $78.2 \pm 6.7$ & $81.6 \pm 7.0$ & -3.01 & $.008^{1}$ \\
\hline Supination in the dominant forearm & $83.4 \pm 5.3$ & $84.9 \pm 4.0$ & -1.30 & .209 \\
\hline Supination in the non-dominant forearm & $84.7 \pm 3.8$ & $86.3 \pm 2.5$ & -1.65 & .118 \\
\hline Dorsal flexion in the dominant wrist joint & $81.8 \pm 5.5$ & $83.0 \pm 10.5$ & -0.61 & .550 \\
\hline Dorsal flexion in the non-dominant wrist joint & $82.9 \pm 7.4$ & $85.1 \pm 8.9$ & -1.07 & .300 \\
\hline Volar flexion in the dominant wrist joint & $83.3 \pm 9.2$ & $84.0 \pm 4.7$ & -0.37 & .717 \\
\hline Volar flexion in the non-dominant wrist joint & $81.7 \pm 6.8$ & $83.6 \pm 7.7$ & -0.95 & .355 \\
\hline Abduction in the dominant hip joint & $49.3 \pm 11.6$ & $52.9 \pm 9.9$ & -1.14 & .269 \\
\hline Abduction in the non-dominant hip joint & $49.4 \pm 10.2$ & $51.1 \pm 7.5$ & -0.59 & .560 \\
\hline Adduction in the dominant hip joint & $21.3 \pm 4.4$ & $24.9 \pm 4.5$ & -3.58 & $.002^{1}$ \\
\hline Adduction in the non-dominant hip joint & $20.7 \pm 4.6$ & $23.7 \pm 3.5$ & -2.65 & $.017^{1}$ \\
\hline Flexion in the dominant knee joint & $157.9 \pm 9.0$ & $159.4 \pm 6.1$ & -1.38 & .184 \\
\hline Flexion in the non-dominant knee joint & $159.1 \pm 6.6$ & $159.4 \pm 6.3$ & -0.54 & .595 \\
\hline Eversion in the dominant subtalar joint & $6.9 \pm 1.7$ & $10.4 \pm 2.3$ & -7.51 & $<.001^{1}$ \\
\hline Eversion in the non-dominant subtalar joint & $7.1 \pm 1.6$ & $10.9 \pm 2.6$ & -6.49 & $<.001^{1}$ \\
\hline Inversion in the dominant subtalar joint & $33.4 \pm 5.9$ & $37.2 \pm 4.9$ & 3.96 & $<.001^{1}$ \\
\hline Inversion in the non-dominant subtalar joint & $29.9 \pm 5.8$ & $35.2 \pm 5.1$ & -4.04 & $<.001^{1}$ \\
\hline
\end{tabular}

Note. $\quad{ }^{1} t$-test showed significantly different value between before and after match play $(p \leq .05)$.

\section{Discussion}

The purpose of this study was to analyze the influence of match play on shoulder, elbow, wrist, hip, knee, and ankle range of motion in young tennis players. Using a special wearable device, players' workloads were assessed. It was found that the average number of shots in a rally was comparable to the values observed in the present study (Kovalchik \& Reid, 2017).

Our results showed that the internal rotation of the dominant shoulder was significantly lower before the tennis match than that of the non-dominant shoulder. The same observation was made by Stanley et al. (2003). Chandler et al. (1990) measured flexibility in junior elite tennis players and concluded that the decrease of the dominant shoulder's internal rotation was due to an adaptation of the posterior shoulder musculature and the capsular structure of the tennis stroke. No significant difference was found between the dominant and non-dominant arm in the external rotation of the shoulder, which is in line with previous findings (Ellenbecker et al., 2002). After 90 minutes of match play only the values of the external rotation of the non-dominant shoulder increased.

Differences were detected in comparing ROM during elevation in the coronal plane between the dominant and non-dominant shoulders before and after the match. A comparison of ROM of the shoulders showed that the values before and after the match increased in the internal rotation of both shoulder joints. The results are in line with the findings of Schmidt-Wiethoff et al. (2004), who found significant differences in the rotations of both the dominant and non-dominant shoulders in professional tennis players.

Junior tennis players performed approximately $60 \%$ of forehand shots and serves, where those two strokes 
included external, as well as internal, dominant shoulder rotations. High upper arm internal rotation angular velocities developed through extensive range of motion of circumduction (Kibler, 1995), and have been noted to play a similarly positive and substantial role in the development of racquet velocity in serve and forehand strokes (Elliott, Marshall, \& Noffal, 1995). This has been confirmed by the findings of Elliott, Reid, and Crespo (2003), who found that serve shoulder rotation, together with forearm pronation, add as much as $40 \%$ to the final speed of the serve.

Similarly, Kovacs (2006) concluded that tennis players, in comparison to other athletes, have a betterdefined ROM of the internal than the external rotation in the shoulder of the dominant arm. Kovacs states that repetitive and numerous executions of the serve are one of the critical reasons for this phenomenon.

ROM values in the elevation of the arm in the coronal plane of both shoulders, before and after the match, increased. According to functional analysis of the serve, elevation in the coronal plane and external rotation of the shoulder joint are included in the preparatory part of the serve. It can be concluded that optimal match duration and players' stroke performance have a positive effect on ROM in the shoulder joint.

Results of ROM measurements of the elbow show significantly higher values after the match than what was expected following functional analysis. Elbow flexion in tennis strokes is not only incorporated into the serve but also into both baseline shots. The range of flexion in the dominant elbow during the forehand is smaller than that of the backhand, where the dominant hand performs the entire range of motion. Reid, Giblin, and Elliot (2013) analyzed ROM of the elbow and wrist during the serve forward swing phase and found that the changes to the ROM and peak angular velocity of the elbow joint extension, during the forward swing, indicate that the role of elbow extension decreases, rather than becomes more pronounced. The latter is incorporated into serve execution as a concentric type of contraction, which mainly has the function of directing the projectile. The increase in ROM is expected due to the involvement of elbow movements. On the other hand, we can conclude that no differences in the increased range of motion, before and after the match, in the wrist were found. With this, we can indirectly respond to the frequent question of tennis coaches, that the wrist is not actively involved in the performance of tennis strokes.

Young et al. (2014) highlighted adequate hip range of motion values, which are required for the transfer of energy from the lower to the upper extremity, along the kinetic chain. Moreno-Pérez et al. (2016) warn against high loads for tennis players that can negatively influence ROM in the hips, and consequently increase the risk of injury. These findings are similar to the present study, as the research mentioned above also failed to find significant differences in passive hip flexion or extension and abduction between extremities. Normal mobility was measured in both male and female tennis players. An increase of adduction range was observed in both hips (before and after the match). The increase in adduction range, results from both concentric and eccentric muscular contractions due to the large number of directional changes in the lateral direction, submaximal movements and sideward cutting steps, sliding and returning to starting position after forehand and backhand executions on the baseline, in addition to both returns of the serve.

A comparison of the results of the inversion of the subtalar joint between the dominant and non-dominant side, after match play, indicates a higher load on the dominant leg. This leg is used to support both the execution of the forehand and all movements or direction changes, especially those that the player makes to the left. Furthermore, Verstegen (2003) suggests that players' muscles need to be able to generate forces through varying ranges of motion, both from the aspect of highperformance enhancement, as well as injury prevention.

Llana-Belloch, Brizuela, Pérez-Soriano, GarcíaBelenguer, and Crespo (2013) found that sideward cutting movements followed by direction changes are of crucial importance in tennis. They added that this is also influenced by footwear which limits supination and allows tennis players to perform faster sideward cutting movements. Our study has shown, that there is an increase in ROM of the subtalar joint due to numerous direction changes and split-step executions. Ankle function is essential for the positioning and setting of the foot, in addition to affecting the player's movement acceleration, and deceleration.

There are several limitations to this study that should be considered. First, due to limitations in the measurement features of the wrist monitor, we were unable to include data on the number of shots during a match, the average number of shots during a rally, and wrist speed during the time of acceleration. As discussed earlier, the load on the observed parts of the body varied during different strokes, which indirectly affected the changes in the range of motion in individual joints. Additionally, we were unable to measure the distance covered or the number of directional changes during a rally.

Secondly, due to all test tennis matches being played on the same day, outside temperatures were not monitored.

Thirdly, the somewhat small sample size was attributed to the fact that only young tennis players 
were included in the study and their participation was voluntary.

In the future, optimal values of ROM in individual joints should also be evaluated more accurately for other young athletes.

\section{Conclusions}

Bilateral measurements of internal rotation in the shoulder joint, forearm pronation, and inversion of the subtalar joints before match play, and of elevation of the arm in the coronal plane, forearm pronation after match play, have identified significant differences between extremities. In the case of junior male and female tennis players, a 90-minute match positively affected ROM values, especially in those motions that were part of tennis movement patterns and shots. Junior tennis players can, with adequate warm-up and preparation, hold or even slightly increase ROM in certain joints, especially if the match is followed by appropriate stretching. Our study suggests that matches lasting up to an hour and a half do not have a negative impact on young athletes.

\section{Acknowledgments}

The authors wish to thank all of the tennis players for participating in this study.

\section{Conflict of interest}

There were no conflicts of interest.

\section{References}

Chandler, T. J., Kibler, W. B., Uhl, T. L., Wooten, B., Kiser, A., \& Stone, E. (1990). Flexibility comparisons of elite junior tennis players to other athletes. American Journal Sports Medicine, 18, 134-136.

Chiang, C.-C., Hsu, C.-C., Chiang, J.-Y., Chang, W.-C., \& Tsai, J.-C. (2016). Flexibility of internal and external glenohumeral rotation of junior female tennis players and its correlation with performance ranking. Journal of Physical Therapy Science, 28, 3296-3299.

Ellenbecker, T. S., \& Roetert, E. P. (2003). Age specific isokinetic glenohumeral internal and external rotation strength in elite junior tennis players. Journal of Science and Medicine in Sport, 6, 63-70.

Ellenbecker, T. S., Roetert, E. P., Bailie, D. S., Davies, G. J., \& Brown, S. W. (2002). Glenohumeral joint total rotation range of motion in elite tennis players and baseball pitchers. Medicine \& Science in Sports \& Exercise, 34, 2052-2056.

Ellenbecker, T. S., Roetert, E. P., Piorkowski, P. A., \& Schulz, D. A. (1996). Glenohumeral joint internal and external rotation range of motion in elite junior tennis players. Journal of Orthopaedic \& Sports Physical Therapy, 24, 336-341.

Elliott, B. C., Marshall, R. N., \& Noffal, G. J. (1995). Contributions of upper limb segment rotations during the power serve in tennis. Journal of Applied Biomechanics, 11, 433-442.

Elliott, B., Reid, M., \& Crespo, M. (Eds.). (2003). ITF biomechanics of advanced tennis. London, United Kingdom: International Tennis Federation.

Gillet, B., Begon, M., Diger, M., Berger-Vachon, C., \& Rogowski, I. (2018). Shoulder range of motion and strength in young competitive tennis players with and without history of shoulder problems. Physical Therapy in Sport, 31, 22-28.

Gillet, B., Begon, M., Sevrez, V., Berger-Vachon, C., \& Rogowski, I. (2017). Adaptive alterations in shoulder range of motion and strength in young tennis players. Journal of Athletic Training, 52, 137-144.

Horger, M. (1989). The reliability of goniometric measurements of active and passive wrist motions. American Journal of Occupational Therapy, 44, 342-348.

Jakovljević, M., \& Hlebš, S. (2017). Meritve gibljivosti sklepov, obsegov in dolžin udov [Measurements of joints flexibility, girths and lengths of limbs]. Ljubljana, Slovenia: University of Ljubljana.

Kibler, W. B. (1995). Biomechanical analysis of the shoulder during tennis activities. Clinics in Sport Medicine, 14, 79-85.

Kibler, W. B., \& Chandler, T. J. (2003). Range of motion in junior tennis players participating in an injury risk modification program. Journal of Science and Medicine in Sport, 6, 51-62.

Kibler, W. B., Chandler, T. J., Livingston, B. P., \& Roetert, E. P. (1996). Shoulder range of motion in elite tennis players. American Journal of Sports Medicine, 24, 279-285.

Knudson, D. V. (2007). Fundamentals of biomechanics. New York, NY: Springer.

Kos, M., \& Kramberger, I. (2017). A wearable device and system for movement and biometric data acquisition for sports applications. IEEE Access, 5, 6411-6420.

Kovacs, M. S. (2006). Applied physiology of tennis performance. British Journal of Sports Medicine, 40, 381-386.

Kovalchik, S. A., \& Reid, M. (2017). Comparing matchplay characteristics and physical demands of junior and professional tennis athletes in the era of big data. Journal of Sports Science and Medicine, 16, 489-497.

Llana-Belloch, S., Brizuela, G., Pérez-Soriano, P., GarcíaBelenguer, A. C., \& Crespo, M. (2013). Supination control increases performance in sideward cutting movements in tennis. Sports Biomechanics, 12, 38-47.

Martin, C., Kulpa, R., Ezanno, F., Delamarche, P., \& Bideau, B. (2016). Influence of playing a prolonged tennis match on shoulder internal range of motion. American Journal of Sports Medicine, 44, 2147-2151.

Moreno-Pérez, V., Ayala, F., Fernandez-Fernandez, J., \& Vera-Garcia, F. J. (2016). Descriptive profile of hip range 
of motion in elite tennis players. Physical Therapy in Sport, 19, 43-48.

Moreno-Pérez, V., Moreside, J., Barbado, D., \& Vera-Garcia, F. J. (2015). Comparison of shoulder rotation range of motion in professional tennis players with and without history of shoulder pain. Manual Therapy, 20, 313-318.

Norkin, C. C., \& White, D. J. (2016). Measurement of joint motion: A guide to goniometry (5th ed.). Philadelphia, PA: F. A. Davis Company.

Reid, M., Crespo, M., Santilli, L., Miley, D., \& Dimmock, J. (2007). The importance of the International Tennis Federation's junior boys' circuit in the development of professional tennis players. Journal of Sports Sciences, 25, 667-672.

Reid, M., Giblin, G., \& Elliott, B. (2013). Effect of a common task constraint on the body, racket and ball kinematics of the elite junior tennis serve. Sports Biomechanics, 12, $15-22$.

Reid, M., \& Morris, C. (2011). Ranking benchmarks of top 100 players in men's professional tennis. European Journal of Sport Science, 13, 350-355.

Riddle, D. L., Rothstein, J. M., \& Lamb, R. L. (1987). Goniometric reliability in a clinical setting. Shoulder measurements. Physical Therapy, 67, 668-673.

Roetert, E. P., Kovacs, M., Knudson, D., \& Groppel, J. L. (2009). Biomechanics of the tennis groundstrokes: Implications for strength training. Strength and Conditioning Journal, 31, 41-49.

Roetert, E. P., McCormick, T. J., Brown, S. W., \& Ellenbecker, T. S. (1996). Relationship between isokinetic and functional trunk strength in elite junior tennis players. Isokinetics and Exercise Science, 6, 15-20.

Rothstein, J. M., Miller, P. J., \& Roettger, R. F. (1983). Goniometric reliability in a clinical setting. Elbow and knee measurements. Physical Therapy, 63, 1611-1615.

Schmidt-Wiethoff, R., Rapp, W., Mauch, F., Schneider, T., \& Appell, H. J. (2004). Shoulder rotation characteristics in professional tennis players. International Journal of Sports Medicine, 25, 154-158.

Shellock, F. G., \& Prentice, W. E. (1985). Warming-up and stretching for improved physical performance and prevention of sports-related injuries. Sports Medicine, 2, 267-278.

Silva, R. T., Gracitelli, G. C., Saccol, M. F., Laurino, C. F., Silva, A. C., \& Braga-Silva, J. L. (2006). Shoulder strength profile in elite junior tennis players: Horizontal adduction and abduction isokinetic evaluation. British Journal of Sports Medicine, 40, 513-517.

Stanley, A., McGann, R., Hall, J., McKenna, L., \& Brigfa, K. N. (2003). Shoulder strength and range of motion in female amateur-league tennis players. Journal of Orthopaedic \& Sports Physical Therapy, 34, 402-409.

Vad, V. B., Gebeh, A., Dines, D., Altchek, D., \& Norris, B. (2003). Hip and shoulder internal rotation range of motion deficits in professional tennis players. Journal of Science and Medicine in Sport, 6, 71-75.

Verstegen, M. (2003). Developing strength. In M. M. Reid, A. Quinn, \& M. Crespo (Eds.), ITF strength and conditioning for tennis (pp. 113-135). London, United Kingdom: International Tennis Federation.

Watkins, M., Riddle, D., \& Lamb, R. (1991). Reliability of goniometric measurements and visual estimates of knee range of motion obtained in a clinical setting. Physical Therapy, 71, 90-97.

Weber, K. (2001). Demand profile and training of running speed in elite tennis. In M. Crespo, M. Reid, \& D. Miley (Eds.), Applied sports science for high performance tennis (pp. 41-48). London, United Kingdom: International Tennis Federation.

Young, S. W., Dakic, J., Stroia, K., Nguyen, M. L., Harris, A. H. S., \& Safran, M. R. (2014). Hip range of motion and association with injury in female professional tennis players. American Journal of Sports Medicine, 42, 2654-2658. 